\title{
Crystal structure of $\mathrm{NiFe}(\mathrm{CO})_{5}$ (tri(pyridylmethyl)azaphosphatrane): a synthetic mimic of the NiFe hydrogenase active site incorporating a pendant pyridine base
}

Natwara Sutthirat, Joseph W. Ziller, Jenny Y. Yang and Zachary Thammavongsy*

Table S1. List of NiFe complexes with Ni-Fe bond distances.

\begin{tabular}{|l|c|l|}
\hline \multicolumn{1}{|c|}{ CSD refcodes } & Ni-Fe distance $(\boldsymbol{A})$ & \multicolumn{1}{c|}{ Ref } \\
\hline FANHEK & 2.621 & 1 (Song et al., 2017) \\
\hline FANHEK01 & 2.614 & 1 (Song et al., 2017) \\
\hline FANGUZ & 2.460 & 1 (Song et al., 2017) \\
\hline FANHIO & 2.650 & 1 (Song et al., 2017) \\
\hline FANHUA & 2.660 & 1 (Song et al., 2017) \\
\hline LAZWEP & 2.467 & 2 (Zhu et al., 2005) \\
\hline SUQQOL & 2.613 & 3 (Barton et al., 2009) \\
\hline UCUXOH & 2.684 & 4 (Carroll et al., 2011) \\
\hline UCUXUN & 2.596 & 4 (Carroll et al., 2011) \\
\hline UQAJAZ & 2.550 & 5 (Manor \& Rauchfuss, 2013) \\
\hline YOKWIE & 2.447 & 6 (Walther et al., 1995) \\
\hline
\end{tabular}


Table S2. List of NiFe complexes with Ni-Fe bond distances.

\begin{tabular}{|l|c|l|}
\hline \multicolumn{1}{|c|}{ CSD refcodes } & Ni-Fe distance $(\boldsymbol{A})$ & \multicolumn{1}{c|}{ Ref } \\
\hline EJUSEJ & 2.572 & 7 (Sun et al., 2016) \\
\hline EJUSUZ & $3.164^{*}$ & 7 (Sun et al., 2016) \\
\hline FOTKOP & 3.296 & 8 (Tanino et al., 2009) \\
\hline QEKLAT & 2.800 & 9 (Liaw et al., 2000) \\
\hline FANHUA & 2.660 & 1 (Song et al., 2017) \\
\hline
\end{tabular}

- Average of two structures in the unit cell.

\section{Reference:}

1 Song, L.-C., Lu, Y., Zhu, L. \& Li, Q.-L. (2017). Organometallics 36, 750-760.

2 Zhu, W., Marr, A. C., Wang, Q., Neese, F., Spencer, D. J. E., Blake, A. J., Cooke, P. A., Wilson, C. \& Schröder, M. (2005). 102, 18280-18285.

3 Barton, B. E., Whaley, C. M., Rauchfuss, T. B. \& Gray, D. L. (2009). Journal of the American Chemical Society 131, 6942-6943.

4 Carroll, M. E., Barton, B. E., Gray, D. L., Mack, A. E. \& Rauchfuss, T. B. (2011). Inorganic Chemistry 50, 9554-9563.

5 Manor, B. C. \& Rauchfuss, T. B. (2013). Journal of the American Chemical Society 135, 11895-11900.

6 Walther, D., Geßler, S. \& Sieler, J. (1995). Zeitschrift für anorganische und allgemeine Chemie 621, 635-639.

7 Sun, P., Yang, D., Li, Y., Zhang, Y., Su, L., Wang, B. \& Qu, J. (2016). Organometallics 35, 751-757.

8 Tanino, S., Li, Z., Ohki, Y. \& Tatsumi, K. (2009). Inorganic Chemistry 48, 2358-2360.

9 Liaw, W.-F., Chiang, C.-Y., Lee, G.-H., Peng, S.-M., Lai, C.-H. \& Darensbourg, M. Y. (2000). Inorganic Chemistry 39, 480-484. 